\title{
Ultrasound Features of the Formation of the Maternal-Placental Complex in Cases of Hyperandrogenism as Observed during the First and Second Trimesters of Pregnancy
}

\author{
${ }^{1}$ Malysheva Olga, ${ }^{2}$ Toropkina Elena, ${ }^{3}$ Agarkova Luybov, ${ }^{4}$ Bukharina Irina, ${ }^{5}$ Logvinov Sergey
}

\begin{abstract}
An excess of maternal androgen adversely affects the implantation process and contributes to uterine circulatory disturbances and myometrium vessel sclerosis. Placental insufficiency is typically diagnosed at the end of the second trimester of pregnancy or at the beginning of the third trimester, when the treatment is not effective.

Many researchers have attempted to use early sonographic diagnostics to identify placental failure in various pathological states. Nevertheless, the peculiarities of the formation of the fetal-placental complex during the first and second trimesters are still undefined. In this study, we traced the process of placental formation under conditions of excess maternal androgen using ultrasound and Dopplerometry. Morphological examinations of the chorion and placental tissue were undertaken to compare any changes.

Unfavorable chorion locations, heterogeneous chorion tissue structures, and marginal sinus dilatations were revealed by ultrasound. According to a Dopplerometric investigation, a statistically significant increase in peripheral vessel resistance in cases of hyperandrogenism was identified compared with healthy pregnancies. The negative influence of hyperandrogenism on placental formation was confirmed by morphological data (including the presence of immature decidual cells, defective villi that covered the epithelium, and the dissociative development of cotyledons).
\end{abstract}

Keywords: Placenta, Hyperandrogenism, Ultrasound examination, Dopplerometry, Morphology.

How to cite this article: Olga M, Elena $T$, Luybov A, Irina B, Sergey $L$. Ultrasound Features of the Formation of the Maternalplacental Complex in Cases of Hyperandrogenism as Observed

\footnotetext{
${ }^{1}$ Researcher, ${ }^{2,3,5} \mathrm{Head},{ }^{4}$ Scientific Secretary

1,3,4 Federal State Budgetary Establishment Research Institution for Obstetrics, Gynecology and Perinatology, Siberian Branch of Russian Academy of Medical Science, Lazo Street, Tomsk Russia

${ }^{2}$ Department of Ultrasound Diagnostics, Federal State Budgetary Establishment Research Institution for Obstetrics, Gynecology and Perinatology, Siberian Branch of Russian Academy of Medical Science, Lazo Street, Tomsk, Russia

${ }^{5}$ Department of Perinatology, Federal State Budgetary Establishment Research Institution for Obstetrics, Gynecology and Perinatology, Siberian Branch of Russian Academy of Medical Science, Lazo Street, Tomsk, Russia
}

Corresponding Author: Malysheva Olga, Researcher 634055, 17, Academicheskiy Prospect, Apt 139, Tomsk, Russia Phone: 89059919590, e-mail: ogm.201@yandex.ru during the First and Second Trimesters of Pregnancy. Donald School J Ultrasound Obstet Gynecol 2014;8(2):149-154.

Source of support: Nil

Conflict of interest: None

\section{INTRODUCTION}

Hyperandrogenism in women is a widespread clinical condition that leads to disorders in reproductive function. A pregnancy with hyperandrogenism is a high-risk pregnancy. ${ }^{4,6,8,34}$ The most frequent complications of such a pregnancy are miscarriage and placental dysfunction. Nevertheless, obstetricians and gynecologists have not agreed upon the need for treatment or the methods of treatment for hyperandrogenism in pregnant women. ${ }^{23,24}$ In connection with this disagreement, studies of the influence of excess maternal androgen on the development and function of the maternal-placental complex are unclear.

Some studies have shown that hyperandrogenism adversely affects implantation and contributes to circulatory disturbances and sclerosis of myometrium vessels. ${ }^{30,32,33}$ However, the changes in the maternal-placental complex during the first and second trimesters have yet to be described. Morphological data describing the early stages of the formation and function of the placenta (the first 12 weeks) cannot be found in the literature.

The formation of the villous chorion and the placenta occurs in a strict temporal sequence. An imbalance in this process may lead to placental dysfunction and intrauterine fetal growth restrictions. ${ }^{26,34}$ In a normal state, the formation of the placenta is complete by the end of the first trimester. At that time, the placenta is protected by the villous chorion and the decidual membrane, which function as an endocrine organ and participate in gas diffusion and nutritional exchange. By the end of the second trimester, the villous tree is a system in which villi are intermediately differentiating and terminal villi are initially generated. The gestational reconstruction of myometrial segments of the maternal and placental arteries also occurs during the second trimester. ${ }^{1,39}$

Disturbances at any stage of gestation as a result of hyperandrogenism may lead to placental dysfunction with nonspecific morphological indicators (including the development of dissociated villi, premature placental maturation, excessive 
fibrinoid deposits, calcinosis, and increased syncytial knots). Some studies have shown decreases in fetal and placental weights because of excess maternal androgen. ${ }^{24,36,38}$

\section{MATERIALS AND METHODS}

The aims of this study were to estimate the state of the fetal-placental complex by Dopplerometry and ultrasound during the first and second trimesters of pregnancy and to compare these data with morphological changes in the placenta. The study was performed in the Research Institute for Obstetrics, Gynecology and Perinatology (Tomsk, Russia) between 2007 and 2011. Women selected for the study had hyperandrogenism (increased level of DHEA, androstenedione, free testosterone, stigmata, BMI, the history of PCOS with fulfilled sonographic criteria) that had been diagnosed during the first trimester of pregnancy. Exclusion criteria were: (1) somatic diseases, (2) sexual diseases and (3) rhesus incompatibility.

For the study, 80 pregnant women at 10 to 12 weeks of gestation (group I) were divided into two subgroups:

- IA: The main group $(\mathrm{n}=42)$ - pregnant women with hyperandrogenism.

- IB: The control group $(\mathrm{n}=38)$ - healthy pregnant women with uncomplicated pregnancies.

Group II consisted of 52 pregnant women at 20 to 22 weeks of gestation, and this group was also divided into two subgroups:

- IIA: The main group $(\mathrm{n}=26)$ - pregnant women with hyperandrogenism.

- IIB: The control group $(\mathrm{n}=26)$ - healthy pregnant women with uncomplicated pregnancies.

Measurements of crown-rump length, heart rate, nuchal space (and the presence of translucency), and nasal bones were performed by ultrasound during the first trimester. The localization, thickness, and structure of the chorion were also estimated..$^{12,17,18}$ Fetometry, measurements of the quantity of amniotic fluid present, and placentometry were performed by ultrasound during the second trimester. The echostructure, location, and thickness of the placenta as well as the state of the intervillous space, the presence of calcification, and the presence of additional placental lobes were described. ${ }^{13}$

The systolic/diastolic velocity ratio, resistance index, and pulsatility index were calculated by Dopplerometry. The maternal-placental blood flow was estimated in both uterine arteries. To measure the fetal-placental blood flow, measurements were taken in both umbilical cord arteries. ${ }^{10,11,20,21,37}$

A histological examination was also performed. The chorion and decidual membrane, obtained after the curettage of the uterine cavities of women undergoing artificial abortions at 10 to 12 weeks of gestation, were examined. Placentas received after therapeutic abortions at 20 to 22 weeks of gestation were also examined. The degree of decidual cell maturation was estimated from histologic specimens. The specific volumes of the basal lamina, the chorial lamina, the intervillous space, fibrinoids, the villous stroma, the placental vessels, the chorial epithelium, syncytial knots, and the calcinates were calculated.

\section{Statistical Analysis}

Data are expressed as mean $\pm \mathrm{SD}$ or as the median (interquartile range). Mann-Whitney nonparametric criteria were used. The critical level of statistical significance (p) was set to 0.05 . The Fisher test and the chi-squared criterion were used to verify the reliability of differences within the qualitative data. The Spearman rank correlation was also used to estimate the relationships between ultrasound and morphological parameters.

\section{RESULTS}

\section{Clinical and Ultrasound Characteristics of Women in the First Trimester of Pregnancy}

The mean maternal age of test subjects was $26.19 \pm 6.13$ years. No significant differences were detected in the parity rate, number of deliveries, or abortions in the compared groups. A total of 12 (28.6\%) women with hyperandrogenia had miscarriages before 22 weeks, whereas only one woman experienced pregnancy loss in the control group.

No statistical differences were revealed in the localization of the chorion on the anterior or posterior wall of the uterus between the two groups. The placenta previa was detected only in the group with hyperandrogenism, in $8(19 \%)$ cases (Fig. 1). Lateral chorion location was observed in $17(40.5 \%)$ women in the hyperandrogenia group and in two (5.3\%) women in the control group, and marginal chorion location was observed in $9(21.4 \%)$ women in the hyperandrogenia

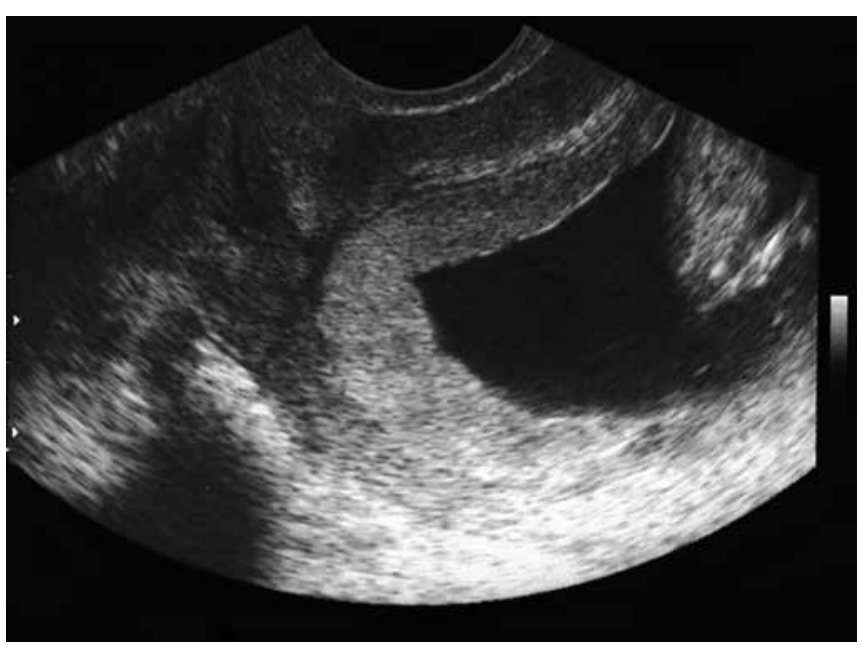

Fig. 1: Placenta previa in case of hyperandrogenism (10-12 weeks) 


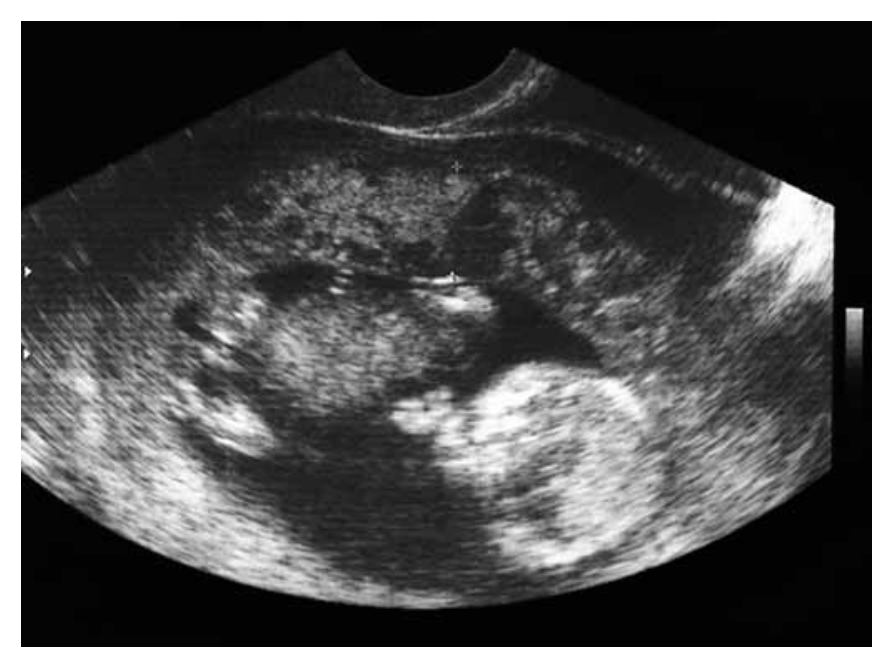

Fig. 2: Heterogeneity of the chorion in case of hyperandrogenism (10-12 weeks)

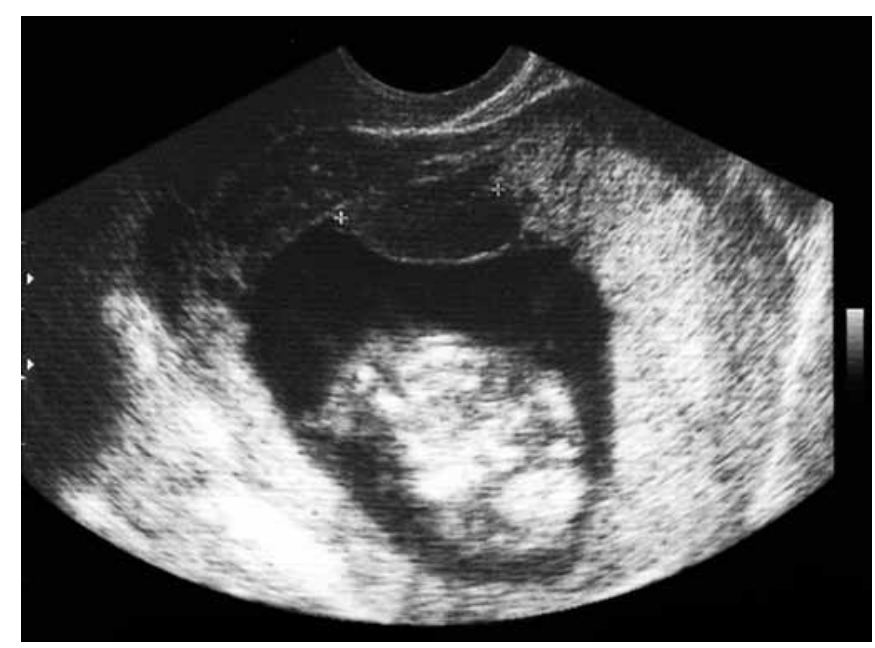

Fig. 3: Dilation of marginal sinus in case of hyperandrogenism (10-12 weeks)

group and in $5(13.2 \%)$ women in the control group. Such chorion presentations are considered to be unfavorable for the blood supply of the developing placenta and may lead to placental dysfunction. ${ }^{15,16,27,41}$

The heterogeneity of the chorion tissue and the alteration of hyperechoic and hypoechoic areas with fuzzy contours were observed in 20 (47.6\%) women with hyperandrogenism-a finding that was statistically different from the control group,

Table 1: Indices of vascular resistance in uterine arteries during the first trimester of pregnancy in cases of hyperandrogenism $(\mathrm{M} \pm \mathrm{m})$

\begin{tabular}{lll}
\hline $\begin{array}{l}\text { Indices of artery flow } \\
\text { velocity waveforms }\end{array}$ & $\begin{array}{l}\text { IA group } \\
(n=42)\end{array}$ & $\begin{array}{l}\text { Control } \\
(n=38)\end{array}$ \\
\hline Resistance index & $0.76 \pm 0.27^{*}$ & $0.64 \pm 0.16$ \\
Pulsatility index & $2.08 \pm 0.59^{*}$ & $1.38 \pm 0.35$ \\
Systolic/diastolic velocity ratio & $4.24 \pm 1.33^{*}$ & $2.66 \pm 0.54$ \\
\hline
\end{tabular}

*Data are statistically significant in comparison with the control group $(p<0.05)$ with 2 (5.3\%) cases (Fig. 2). The presence of hypoechoic structures in the placental parenchyma is considered to result from a blood supply disturbance. However, hyperechoic structures are characterized by the rate of histogenesis, the degree of differentiation of the villous stroma, and the level of compensatory reaction (direct relation). ${ }^{1,39,26,40}$

The sonographic measurements of placental thickness did not reveal any differences between the studied groups. Dilatation of the marginal sinus by more than $7 \mathrm{~mm}$ was observed in seven $(16.7 \%)$ women in the hyperandrogenia group and in one (2.7\%) woman in the control group (Fig. 3). It is believed that the dilatation of the marginal sinus may be the result of a blood outflow disturbance and may lead to intervillous thrombosis. ${ }^{16,17,20,22}$

A statistically significant increase in the resistance index in women with hyperandrogenia in comparison with healthy pregnant women was revealed by Dopplerometry (Table 1).

The mean data for blood flow (resistance index, pulsatility index, systolic/diastolic velocity ratio) in the uterine arteries of pregnant women with hyperandrogenism were significantly higher than in the control group and were also at the upper limit of the measurement. We suspect that these data indicate the discirculatory abnormalities in uterine arteries resulting from maternal underperfusion in case of hyperandrogenism. In the hyperandrogenia group, 30\% of women had the abnormal Doppler patterns of varying degree (in the control group, only $8.3 \%$ of women had such patterns).

The high vascular resistance in uterine arteries contributes to the insufficiency of maternal flow as the main factor driving placental insufficiency. ${ }^{3,5,7,9}$ The majority of the authors believe that an increased resistance in fetoplacental circulation may be the result of the incomplete gestational reconstruction of uterine-placental vessels due to the disturbance of trophoblast invasion. ${ }^{35}$

\section{Morphological Characteristics of the Maternal-placental Complex during the First Trimester of Pregnancy}

The gestational remodeling of spiral arteries was identified in both groups by morphological examination. The presence of immature decidual cells was revealed only in hyperandrogenic placentas, which may be evidence of the insufficient growth of invasive trophoblasts during the initial stage of placentation. The numbers of mesenchymal and immature intermediate villi in the placental stroma were the same in the hyperandrogenia and control groups. Immature and intermediate villi characterize the friable stroma, which consists of fibroblasts and Hofbauer cells, which form a bilayered epithelial covering. However, we identified 
the presence of villi with defective epithelial coverings, where damaged portions were replaced by fibrinoids. A more compact disposition of fibroblasts was also observed in these cases; fetal vessels were surrounded by growing collagen fibers.

A statistically significant decrease in the specific volumes of syncytial knots, which characterized the trophoblast proliferative capability and villus formation, was found in the hyperandrogenia group. An inversely correlated dependence between the proliferation of villi in the epithelium and the level of androgens in the blood was revealed by statistical analysis $(\mathrm{r}=-0.28, \mathrm{p}=0.031)$.

The specific volume of placental vessels was larger in the hyperandrogenia group $(10.5 \pm 1.7 \%)$ than in the control group $(8.5 \pm 2.3 \%), \mathrm{p}>0.05$, which may indicate compensatory changes. ${ }^{1}$ Moreover, the closer location of capillaries to the basal membrane of chorial epithelium and the removal of larger vessels to the peripheral villi were observed in the placentas of hyperandrogenic women. Many erythroblasts were found alongside the erythrocytes in the fetal vessels, which indicates the retardation of blood formation from the gestational norm.

\section{Clinical and Ultrasound Characteristics of Women during the Second Trimester of Pregnancy}

The clinical characteristics of the pregnant women studied (including age and menarche) were the same as those during the first trimester. Gynecological anamnesis in the hyperandrogenia group was compromised by menstrual disorders (oligomenorrhea), abortions, miscarriages, and stillbirths. Current pregnancies were complicated by abortion in 16 (61\%) women with hyperandrogenia.

The fetometric data in both groups were normal for the gestational ages of the fetuses. The detection of placental localization toward the uterine walls revealed an equal frequency of right and left wall localization $(42.3 \%$ in each group). The localization of the placenta toward the interior faces of the uterus was as follows: lateral localization-6 $(23.1 \%)$ in the hyperandrogenia group, $2(7.7 \%)$ in the control group; marginal localization-5 (19.2\%) in the hyperandrogenia group, 3 (11.6\%) in the control group. Low placentation was observed in five (19.2\%) women in the hyperandrogenia group and in four $(15.4 \%)$ women in the control group. Unfavorable placental localization is considered to be a risk factor for placental dysfunction. ${ }^{2,16,17,31}$

An increase in placental thickness above the relative gestational standard was found in 10 (38.5\%) women with hyperandrogenism. This finding was significantly different from the data from the control group, which showed four
Table 2: Indices of vascular resistance in the uterine arteries during the second trimester of pregnancy in cases of hyperandrogenism, $\mathrm{M} \pm \mathrm{m}$

\begin{tabular}{lll}
\hline $\begin{array}{l}\text { Indices of artery flow } \\
\text { velocity waveforms }\end{array}$ & $\begin{array}{l}\text { IA group } \\
(n=26)\end{array}$ & $\begin{array}{l}\text { Control } \\
(n=26)\end{array}$ \\
\hline Resistance index & $0.67 \pm 0.10$ & $0.61 \pm 0.11$ \\
Pulsatility index & $1.53 \pm 0.42$ & $1.14 \pm 0.38$ \\
Systolic/diastolic velocity ratio & $3.15 \pm 1.04$ & $2.54 \pm 0.97$ \\
\hline
\end{tabular}

Table 3: Indices of vascular resistance in the umbilical cord arteries during the second trimester of pregnancy in cases of hyperandrogenism, $\mathrm{M} \pm \mathrm{m}$

\begin{tabular}{lll}
\hline $\begin{array}{l}\text { Indices of artery flow } \\
\text { velocity waveforms }\end{array}$ & $\begin{array}{l}\text { IA group } \\
(n=26)\end{array}$ & $\begin{array}{l}\text { Control } \\
(n=26)\end{array}$ \\
\hline Resistance index & $0.74 \pm 0.23$ & $0.61 \pm 0.11$ \\
Pulsatility index & $1.37 \pm 0.67$ & $1.29 \pm 0.77$ \\
Systolic/diastolic velocity ratio & $3.79 \pm 1.34$ & $3.48 \pm 1.03$ \\
\hline
\end{tabular}

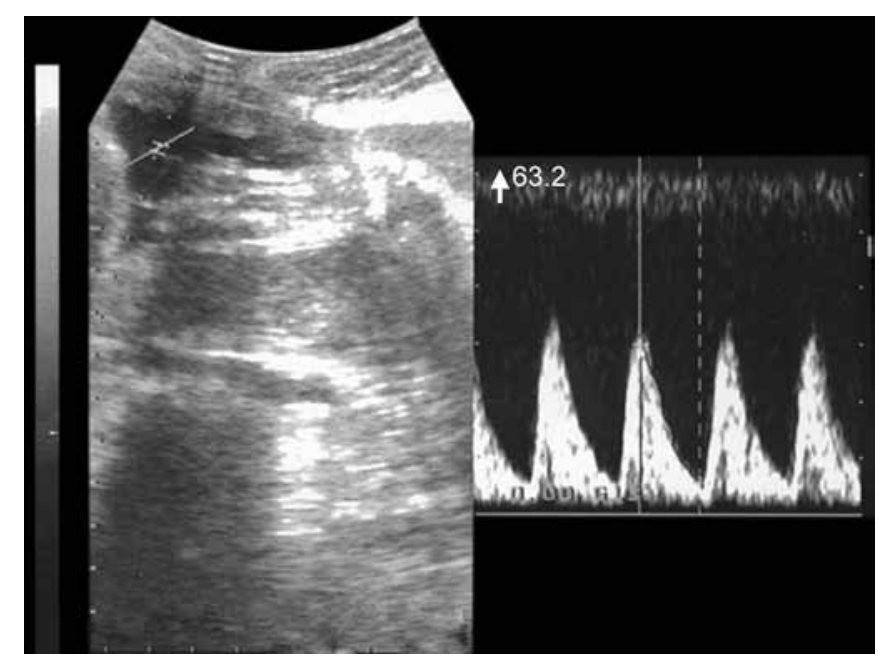

Fig. 4: Abnormal Dopplerometry pattern. Hyperandrogenism (22 weeks)

(15.4\%) women with increased placental thicknesses. According to the literature, an increase in placental thickness is the result of angiomatosis of the intermediate and terminal villi as a compensatory reaction to chronic hypoxia. ${ }^{2,19,25,29}$ Evidently, the placenta works with an increased load to provide adequate oxygen and nutrition transportation.

The placental structure in cases of hyperandrogenism was heterogenic in $14(53.9 \%)$ women [and in six $(23.1 \%)$ women in the control group]. Excess calcium deposits were identified in $46.2 \%$ placentas of women in the hyperandrogenia group. Excess calcium is considered to be unfavorable and is observed in placentas exhibiting premature maturation. ${ }^{1,28,39,40}$

As distinct from healthy pregnant women, six $(23.1 \%)$ hyperandrogenic women had additional placental lobes, three $(11.6 \%)$ had retroplacental hematomas, and six $(23.1 \%)$ had marginal sinus dilatation. Premature placental maturation was found in $12(15.4 \%)$ women with hyperandrogenia and in four $(15.4 \%)$ women in the control group. 
The results of Dopplerometry are presented in Table 2 . The indices of peripheral resistance (resistance index, pulsatility index and systolic/diastolic velocity ratio) were higher in hyperandrogenic women than in healthy pregnant women (Fig. 4). Thus, the indices of arterial flow velocity waveforms in the hyperandrogenia group $(50 \%)$ were significantly higher than those in the control group (30\%). The presence of such changes indicated high vessel resistance that was the result of incomplete trophoblast invasion in the myometrium segments of the spiral arteries.

The estimate of blood flow velocity in the umbilical cord arteries did not reveal the significant differences between the two groups, but the hyperandrogenia group tended to have higher resistance indices (Table 3).

\section{Morphological Characteristics of the Fetal-Placental Complex during the Second Trimester of Pregnancy}

The villus structure of placentas at 20 to 22 weeks includes all types of villi, but their distribution in the hyperandrogenia group was different from their distribution in the control group, thus providing evidence of dissociative cotyledon development. ${ }^{1,14}$

The maximal nearness of capillaries to the syncytial epithelium in terminal villi was observed in the placentas of the hyperandrogenia group and is considered to be the result of the formation of a syncytio-capillary membrane. The fetal vessels in these villi were situated very close together and were filled with mature erythrocytes. Compensatory angiomatosis as a consequence of placental insufficiency was observed in hyperandrogenic placentas. The epithelial cover of the chorion villi was significantly thinned in its main areas. A large number of syncytial knots were observed, which are thought to be the result of compensatory processes of the placenta aimed at maintaining adequate gas exchange.

The specific volumes of intervillous space in cases of hyperandrogenia were lower than those in the control group. Moreover, the prevalence of fibrinoid deposits was twice as high as in the control group (mainly in the marginal zone of the placenta). Loci of thrombosis were found only in the marginal zones of hyperandrogenic placentas. Such changes are due to blood flow disturbances in the intervillous space and lead to an excess of calcium and fibrinoid deposits.

A direct dependence between unfavorable placental presentation and the presence of calcium deposits was revealed by correlation analysis $(r=0.32, p=0.020)$. Direct dependencies were also found between increases in blood flow velocity and the presence of calcium deposits $(\mathrm{r}=0.43$, $p=0.002$ ), increases in the specific volume of fibrinoid deposits $(r=0.32, p=0.02)$, and thrombosis of the intervillous space $(r=0.34, p=0.015)$.

\section{CONCLUSION}

Thus, the primary peculiarities of placentas of hyperandrogenic gravidae during the second trimester are unfavorable presentations, low placentation, heterogenic placental structures, immature placental maturation, an excess of calcium deposits, dilatations of the marginal sinus, and the presence of additional placental lobes. The observed ultrasound changes in placentas were accompanied by histological changes such as dissociative villus development, an increased number of syncytial knots, peripheral trophoblast elements and fetal vessels in the paracentral placental zone, and an excess of fibrinoid and calcium deposits.

\section{REFERENCES}

1. Almasry S, Elfayomy AK. Morphometric analysis of terminal villi and gross morphological changes in the placentae of term idiopathic intrautherine growth restriction. Tissue Cell 2012;44(4):214-219.

2. Alwasel S, Abotalib Z, Osmand C. The breadth of the placental surface but not the length is associated with body size at birth. Placenta 2012;33(8):619-622.

3. Apel-Sarid L, Levy A, Holcberg G, Sheiner E. Term and preterm placental pathologies associated with fetal growth restriction. Arch Gynecol Obstet 2010;282(5):487-492.

4. Azziz R, Nestler JE, Dewailly D. Androgen excess disorders in women: polycystic ovary syndrome and other disorders. 2nd ed. Totowa, New Jersey: Humana Press 2006. p. 349-378.

5. Babovic I, Plesinac S. Doppler examination in the evaluation of outcomes in pregnancies complicated by fetal intrauterine growth retardation — is it enough? Clin Exp Obstet Gynecol 2012;39(2): 222-224.

6. Barbieri RJ. Hyperandrogenic disorders. Clin Obstet Gynec 1990;33(3):640-654.

7. Bolz N, Kalache KD, Slowinski T. Value of Doppler sonography near team: can umbilical and uterine indices in low-risk pregnancies outcome? J Perinat Med 2012;12:1388-1394.

8. Breckwoldt M. Diagnosis and therapy of androgenization. Berlin: Diesbach Verglag 1992. p. 253-267.

9. Bujold E, Effendy M, Girard M, et al. Reproducibility of first trimester three-dimensional placental measurements in the evaluation of early placental insufficiency. J Obstet Gynecol Can 2009;31(12):1144-1148.

10. Campbell S, Black RS, Lees CC, et al. Doppler ultrasound of the maternal uterine arteries: disappearance of abnormal waveforms and relation to birthweight and pregnancy outcome. Acta Obstet Gynecol Scand 2000;79(8):631-634.

11. Carr D, Aitken R, Milne YS. Fetoplacental biometry and umbilical artery Doppler velocimetry in the overished adolescent model of fetal growth restriction. Am J Obstet Gynecol 2012;7:1266-1273.

12. Celen S, Dover N, Goker U. Utility of first trimester ultrasonography before 11 weeks of gestation. Obstet Gyn 2012;34(5): 1344-1354.

13. Chen K, Chen LR. The role of preterm placental calcification in high-risk pregnancy as a predictor of poor uteroplacental blood flow and adwerse pregnancy outcome. Ultrasound Med Biol 2012;38(6):1011-1018.

14. Constantinidi D, Walker M, Milligan N. Pathologic basis of improving the screening utility of 2-dimensional placental morphology ultrasound. Placenta 2012;33(10):845-849. 
15. Devarajan K, Kives S. Placental location and newborn weight. J Obstet Gynec Can 2012;34(4):325-329.

16. Filipov E, Khadzhiev A, Novachkov V, Iakimova A. The relationship of the location of the placenta and the indices of the fetus birth process. Acush Ginekol (Sofiia) 1990;29(2): 15-18.

17. Hata T, Tanaka H, Noguchi J, Nata K. Three-dimensional ultrasound evaluation of the placenta. Placenta 2011;32(2): 105-115.

18. Hsu S, Euerk BD. Ultrasound in pregnancy. Emerg Med Clin North Am 2012;4:849-867.

19. Hutcheon J, McNamara H, Platt RV. Placental weight for gestational age and adverse perinatal outcomes. Obstet Gynecol 2012;11(9):1199-1207.

20. Jauniaux E, Gavriil P, Nicolaides K. Ultrasonographic assessment of early pregnancy complications. Ultrasound and Early Pregnancy. In: Jukovic D, Jauniaux E, editors. London: Parthenon Publishing, 1996, p. 53-65.

21. Jurkovic D, Jauniaux E, Kurjak A, Hustin J, Campbell S, Nicolaides K. Transvaginal color Doppler assessment of the uteroplacental circulation in early pregnancy. Obstet Gynecol 1991;77(3):365-369.

22. Kiserud T. Global reproductive health: is diagnostic ultrasound appropriate technology? Ultrasound Obstet Gynec 2012;40(2): 123-125.

23. Kurtoglu S, Sacrici D, Akin DA. Fetal adrenal suppression due to maternal corticosteroid use: case report. Clin Res Pediatr Endocrin 2011;11(3):160-162.

24. Lee J, Park S, Kim S, Kim MN. Prenatal administration of dexamethasone during early pregnancy negatively affects placental development and function in mice. J Anim Sci 2012; 7:244-248.

25. Lee AG, Bethune M, Hiscorck R. Placental thickness in the second trimester. J Ultrasound Med 2012;32(2):213-218.

26. Lindushka N, DekanS, Messerchmidt A. Placental pathologies in fetal MRI with pathohistological correlation. Placenta 2009; 30(6):555-559.

27. Melchiorre K, Leslic K, Prefumo F, Bhide A. First-trimester uterine artery Doppler indices in the prediction of small-forgestational age pregnancy and intrauterine growth restriction. Ultrasound Obstet Gynec 2009;33(5):524-529.
28. Moran M, Higgins M, Zombori G. Computerised assessment of placental calcification post ultrasound. Ultrasound Obstet Gynec 2012;1:1344-1350.

29. Ortigosa C, Nomura R, Costa V. Fetal venous Doppler in pregnancies with placental disfunction. J Maternal Fetal Neonatal Med 2012;8:6788-6794.

30. Palomba S, Falbo A, Russo T, Tolino A, Orio F, Zullo F. Pregnancy in women with polycystic ovary syndrome: the effect of different phenotypes and features on obstetric and neonatal outcomes. Fertil Steril 2010;94(5):1805-1811.

31. Pooh RK, Kurjak A. 3D/4D sonography moved prenatal diagnosis of fetal anomalies from the second to the first trimester of pregnancy. J Matern Fetal Neonatal Med 2012 May;251(5):433-455.

32. Rabe T. Hyperandrogenism in women. In Rabe T, Grunwald K, Runnebaum B. Gynecological Endocrinology. New York, Springer-Verlad, 2001. p. 517-579.

33. Recabarren S, Sir-Peterman T, Maliquco M, Lobos A. Prenatal exposure to androgens as a factor of fetal programming. Rev Med Chil 2006;134(1):101-108.

34. Robert I. Hyperandrogenic disorders. Clinic Obstet Gynec 1990; 33(3):25-29.

35. Schwartz W, Mandel D, Coletta J. Placental morphologic features and chronic surface vasculature at term are highly correlated with 3-dimensional sonographic measurements at 11 to 14 weeks. J Ultrasound Med 2012;33(2):1277-1282.

36. Sun M, Maliqueo M, Benrick A, Johansson J. Maternal androgen excess reduce placental and fetal weights, increases placental steroidogenesis and leads to long-term health effects in their female offspring. Am J Physiol Endocrin Metab 2012;9:1455-1464.

37. Tutschek B, Tercanli S. Current aspects of obstetric ultrasound in the screening setting. Praxis 2012;14:1473-1480.

38. Usadi R, Legro RS. Reproductive impact of polycystic ovary syndrome. Curr Opin Endocr Diabetes 2012;19(6):505-511.

39. Ventolini G. Conditions assotiated with placental dysfunction. Minerva Gynecol 2011;63(5):459-464.

40. Wallace J, Horgan GW, Bhattacharya S. Placental weight and efficiency in regulation to maternal body mass index and the risk of pregnancy complications. Placenta 2012;33:611-618.

41. Yeniel AO, Ergenoglu A. Effect of placenta previa on fetal growth restriction and stillbirth. Arch Gynecol Obstet 2012;286(2): 295-298. 